\title{
Oral erosive lichen planus with desquamative gingivitis: A case report- Innovative approaches and review of treatment modalities
}

\author{
Rashmi Deshpande Sathe ${ }^{1,{ }^{*}}$, Ragini Motwani ${ }^{2}$, Pushkar Dwivedi ${ }^{3}$, Meenakshi Sood Mahendra ${ }^{4}$, \\ Palak Gangwal Jain ${ }^{5}$
}

\begin{abstract}
${ }^{1}$ Consultant Oral Physician and Radiologist, Dept. of Oral Medicine and Radiology, Vardaan Dental Clinic, Bhopal, Madhya Pradesh, ${ }^{2}$ Private Clinic, Dept. of Oral Pathology and Microbiology, Bhopal, Madhya Pradesh, ${ }^{3}$ Assistant Professor, Dept. of Prosthodontics, People's College of Dental Sciences \& Research Centre, Bhopal, Madhya Pradesh, ${ }^{4}$ General Practitioner, Dept. of Oral Medicine and Radiology, Private Clinic, Rajasthan, ${ }^{5}$ Consultant, Dept. of Oral Medicine and Radiology, The Dental Hub Multispeciality Dental Clinic, Pune, Maharashtra, India

*Corresponding Author: Rashmi Deshpande Sathe

Email: rashmissathe@gmail.com
\end{abstract}

\begin{abstract}
Oral Lichen planus is a common immunological inflammatory mucocutaneous disorder. A 38-year-old female patient reported to the Department of Oral Medicine and Radiology with the chief complaint of burning sensation of oral cavity and bleeding gums since one year. A provisional diagnosis of erosive lichen planus with desquamative gingivitis was given. After initial treatment, drug delivery tray for increased efficacy of drug at local level was considered. Maxillary and mandibular drug delivery trays were made and trimmed upto the level of marginal gingiva. 10mg Prednisolone tablet was crushed and mixed with normal saline and applied on the margins of both the trays. The trays were placed in the oral cavity such that the paste comes in contact with the marginal gingiva. The trays were kept in mouth in same position for 20 minutes. The patient was given instructions to repeat the procedure three times daily and was asked to report after 7 days. After 7 days there was $50 \%$ reduction in gingival erythema and burning sensation was reduced to (VAS 1/10). The patient was kept under periodic follow up since one year and is free of lesions. In the present case report we have explored the use of local drug delivery system for treatment of desquamative gingivitis in OLP and also have reviewed other new treatment modalities. Continuous development in management protocol for OLP is required due to recent in $\neg$ crease in the incidence of malignant transformation rate even in the non-risk population group.
\end{abstract}

Keywords: Erosive OLP, Desquamative gingivitis, Local drug delivery trays, Newer treatment modalities.

\section{Introduction}

Oral Lichen planus is categorized as mucocutaneous disorder that is etiologically varied. The different aspects of this disease includes autoimmunity and inflammation. The lesions of OLP have a variety of appearances ranging from keratotic (reticular or plaque like) to erythematous and ulcerative. ${ }^{1,2}$ Eisen (2005) defined OLP as a relatively common chronic inflammatory disorder affecting squamous epithelia. ${ }^{3}$ Histologically, a prominent $\mathrm{T}$ - lymphocyte response in the immediate underlying connective tissue can be seen. ${ }^{4}$ Sir Erasmus Wilson, a British Physician, ${ }^{5}$ was first to describe Lichen Planus and named the condition leichen (Lichen) Planus in 1869. In literature different prevalence figures for oral lichen planus have been reported ranging from $0.5-2.2 \%$. A higher proportion of women are affected as compared to men. The mean age at the time of diagnosis is approximately 55 years. ${ }^{6,7}$

\section{Case Report}

A 38-year-old female patient reported to the Department of Oral Medicine and Radiology. Her chief complaint was burning sensation of oral cavity and bleeding gums since one year. Burning sensation was insidious in onset, continuous, intensity was severe. It aggravated on having spicy food substances and there was absence of any relieving factors. On taking a complete history the patient also complained of generalized body itching. Past dental history revealed that she had undergone scaling 8 months back. Her medical history revealed that the patient was under antihypertensive medication since 1 year. There was no significant family history. She was married since 18 years having two children. She had a mixed diet. No history of any deleterious habits. She brushed her teeth once daily in horizontal manner using toothbrush and toothpaste. General physical examination revealed that she was moderately built and nourished. The patient appeared depressed and anxious and it was also confirmed on questioning her family members.

On intra-oral examination solitary mixed red and white lesion, irregular in shape, measuring about $2 \times 3$ $\mathrm{cm}$ was present adjacent to left retromolar area. On right buccal mucosa diffuse white patch measuring 2.5 $\times 1.5$ was seen with annular pattern and interlacing striae. The mucosal region, surrounding buccal mucosa was normal. Inspectory findings were confirmed on palpation. Burning sensation as recorded on VAS was $8 / 10$. On examination of hard tissue, there was absence of any caries, missing tooth, root stump or sharp tooth.

Generalised inflammation of marginal and papillary gingiva was present. Grade I calculus and plaque was present and oral hygiene was fair.

Accordingly, a provisional diagnosis of erosive lichen planus on left buccal mucosa and reticular lichen planus on right buccal mucosa with desquamative gingivitis was given. 
Following investigations were advisedorthopantomogram (OPG), complete hemogram, blood sugar, cytosmear, and incisional biopsy from left buccal mucosa. All laboratory findings were within normal limits, and biopsy confirmed as Lichen planus. Psychiatric consultaion and counselling was done for the patient.

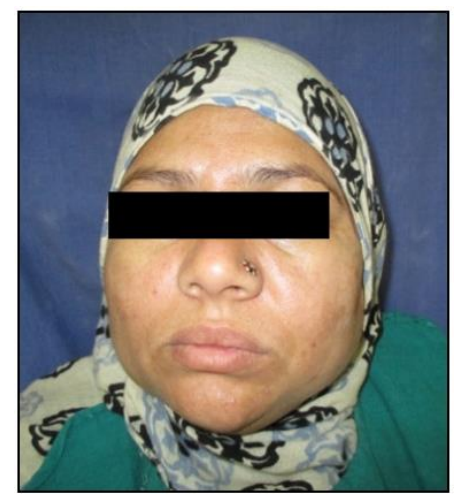

Fig. 1: 38 year old female patient

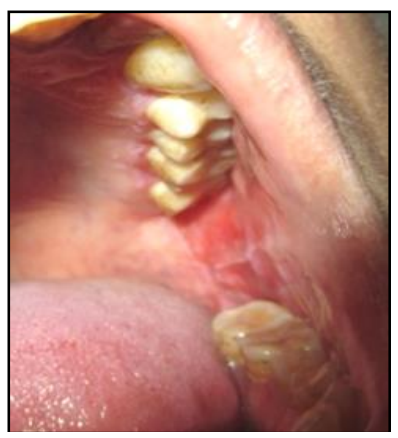

Fig. 2: Left buccal mucosa erosive LP

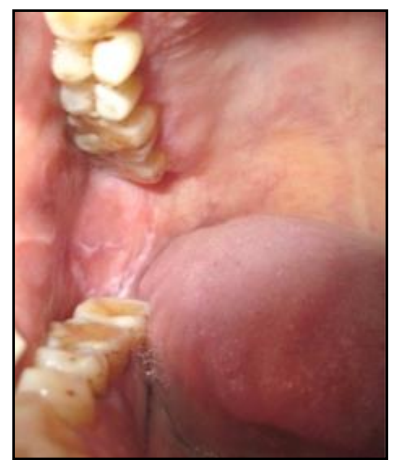

Fig. 3: Right buccal mucosa reticular LP
Initial treatment included:

1. Tab levamisole (Vermisol) $150 \mathrm{mg}$ once daily for 3 days (for 3 weeks)

2. Tab prednisolone (Wysolone) $10 \mathrm{mg} 2$ times a day for 7 days (swish and swallow)

3. benzydamine oral rinse $0.15 \%$ (Tantum oral rinse) (2-3 times daily for 7 days)

Patient was recalled after 7 days. On second visit after 7 days, patient reported with 50\% reduction in burning sensation (VAS 4/10) and the dose of prednisolone was tapered to $10 \mathrm{mg}$ OD. On third visit after 15 days patient reported with complete reduction of burning sensation. On fourth visit after one month lesions on left buccal mucosa had healed and for remaining white lesions, triamcinolone acetonide $0.1 \%$ (caziq) was prescribed for topical application. But the desquamative lesions on gingiva were still present. Patient was kept under observation.

After one month fresh eruptions were present on the upper labial mucosa in the region of 23. Desquamative lesions on gingiva were present causing burning sensation and difficulty in eating. Burning sensation as recorded on VAS scale was 5/10.

Drug delivery tray for increased efficacy of drug at local level was considered. Maxillary and mandibular drug delivery trays were made and trimmed upto the level of marginal gingiva. 10mg Prednisolone tablet was crushed and mixed with normal saline and applied on the margins of both the trays. The trays were placed in the oral cavity such that the paste comes in contact with the marginal gingiva. The trays were kept in mouth in same position for 20 minutes. After which the patient was asked to rinse. The patient was given instructions to repeat the procedure three times daily and was asked to report after 7 days. After 7 days there was 50\% reduction in gingival erythema and burning sensation was reduced to (VAS 1/10). The dose of prednisolone was tapered to $10 \mathrm{mg}$ crushed tablet OD following the same procedure. The patient was recalled after 15 days and there was a complete remission of gingival lesions and burning sensation. The patient was kept under periodic follow up since one year and is free of lesions.
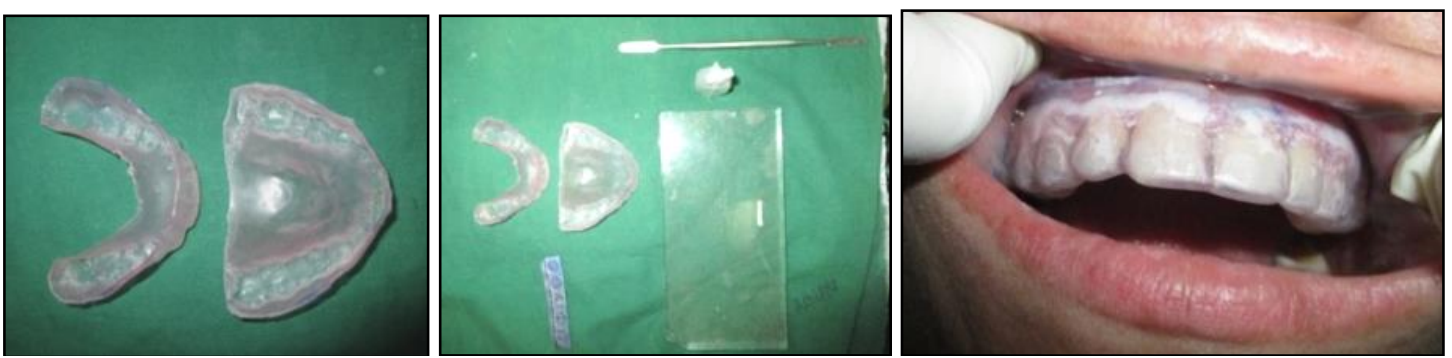

Fig. 4, 5 and 6: Maxillary and mandibular drug delivery trays 


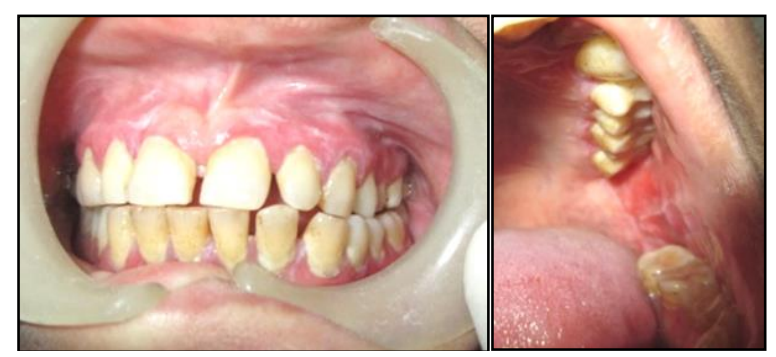

Fig. 7: Pre treatment photographs

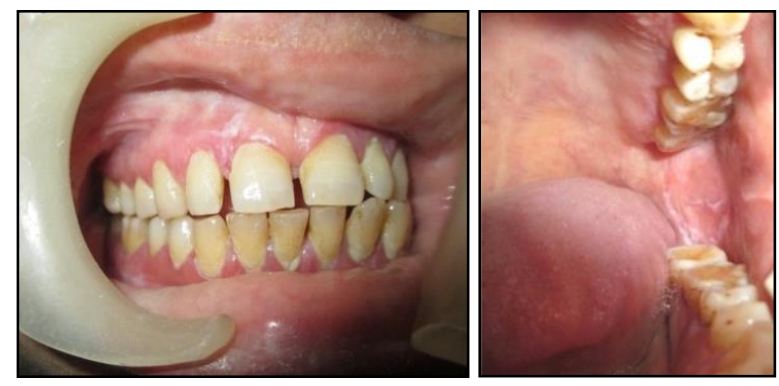

Fig. 8: Post treatment photographs

\section{Discussion}

Oral lichen planus (OLP) tends to be quite resistant to routine therapeutical approach and its persistent nature leads to a lot of mental trauma to the patients. The occurance of oral form of Lichen Planus is more common than the cutaneous form. ${ }^{8}$ Thibierge, in 1885 was first to describe the oral lesions systematically. ${ }^{9}$

Wickham in 1895 described the characteristic whitish striae also known as Wickham's striae. They were punctuations and thin interlacing lines present on the flat surfaced papules. Andreasen in 1968 described 6 forms of oral lichen planus and also stated that, reticular type of lichen planus is most common, and more than one type of oral lesion may occur at the same time. The review of literature shows that about $50 \%$ of the patients with skin lesions have oral lesions, whereas about $25 \%$ of all Lichen Planus patients have only oral lesions. ${ }^{10}$

Aetiology of Lichen Planus has been largely postulated by the expression of the cell mediated arm of the immune system through $\mathrm{T}$ - lymphocyte cytotoxicity directed against antigens expressed by the basal cell layer. In past etiologic considerations in regard to Lichen Planus covered a wide range of possibilities like- 1) Traumatic 2) specific bacterial 3) syphilitic 4) parasitic 5) viral 6) mycotic 7) allergic 8) toxic 9) nervous or neurogenic 10) Hereditary 11) psychosomatic. Contributory factors implicated have been overwork, mouth irritation, and smoking. ${ }^{11}$

To establish a clinical diagnosis of OLP, reticular or papular textures have to be present. OLP confined to the gingiva may be entirely erythematous, or ulcerative areas are present, and this type of lesion has to be confirmed by histopathologic examination. ${ }^{12,13}$

The white and red components of the lesion can be a part of the following textures.

\section{Reticulum}

2. Papules

3. Plaque-like

4. Bullous

5. Erythematous

6. Ulcerative ${ }^{14}$

Histological findings include hyper parakeratosis or hyper ortho-keratosis with thickening of the granular cell layer. There is a presence intracellular edema and acanthosis of the spinous cells. There is a characteristic presence of a "saw tooth" rete pegs. Sometimes there is band - like subepithellial mononuclear infiltrate consisting of $\mathrm{T}$ - cells and histiocytes. sometimes of colloid (civatte, hyaline, cytoid) bodies may also be seen. Direct immunofluoroscence may be useful in differentiating Lichen Planus from lupus erythematosus or pemphigoid. ${ }^{15}$

Differential diagnosis of lichen planus must consider the range of other lichenoid lesions (eg, druginduced lesions, contact mercury hypersensitivity, erythema multiforme, lupus erythematosus, and graftversus-host reaction), as well as leukoplakia, squamous cell carcinoma, mucous membrane pemphigoid, and candidiasis. A detailed history of the clinical appearance and distribution of the lesions is very helpful. ${ }^{16}$

There may be worsening of lesions of OLP due to mechanical trauma (Koebner phenomenon). Oral lichenoid lesions may also be induced from calculus deposits, sharp teeth, rough surfaces of dental restorations or prostheses, cheek or tongue biting and oral surgical procedures. ${ }^{17}$

The presence of dental plaque and calculus results in aggrevating gingival lichen planus. Thus poor oral hygiene results in increased incidence of erythematous and erosive gingival lesions. ${ }^{18}$ Hence Oral lichen planus patients should be instructed in following thorough oral hygiene methods.

OLP associated with chronic oral $C$. albicans infection is recognized as an oral cancer risk factor. Hence, candidal culture or smear should be undertaken periodically and $C$. albicans superinfection should be controlled with topical polyene or azole antimycotics. Patient should be advised to refrain from other deleterious habits. Some studies have shown an association between OLP, hepatitis $\mathrm{C}$ virus (HCV) infection, chronic active hepatitis and primary biliary cirrhosis. ${ }^{19}$

\section{Treatment modalities for OLP}

Treatment should be directed after considering the degree of clinical involvement, clinical type of lesions, the patient's symptoms, and age. There is no cure for OLP, and treatment strategies are essentially designed to limit progression and reduce exacerbations and relive symptoms. Patients must understand that their illness is chronic, may wax and wane over time, and that palliative /support care is the goal. Reticular lesions that 
are asymptomatic generally require no therapy but only observation for change. Our basic goal should be to treat the erosive lesions, eliminate them and periodically observe them so as to decrease the risk of malignant transformation. ${ }^{20}$

Corticosteroids have been the mainstay of management of OLP. They can be used as a

1. Topical: The mild to moderate symptomatic lesions are treated using topical corticosteroids. Eg. Triamcinolone acetonide $0.1 \%, 0.05 \%$ flucinonide, $0.025 \%$ clobetasol propionate etc. Patients are instructed to apply a thin layer of the prescribed topical corticosteroid upto 3 times a day for 2 weeks. $^{21}$

2. Intralesional: Local injection of up to 0.2 to 0.4 $\mathrm{ml}$ of triamcinolone acetonide containing $10 \mathrm{mg} / \mathrm{ml}$ is used to treat persistent localized lesions.
3. Systemic therapy of corticosteroids should be given as a single high-dose preferably in morning for a short period of time. This is done to maximize therapeutic effect while minimizing side effects. A single daily morning dose of 40 to $80 \mathrm{mg}$ of prednisone is prescribed for no more than 10 days. It carries a risk of suppression of HPA axis. Another approach to reduce the amount of total prednisolone dose, is to concurrently prescribe a steroid-sparing agent such as the immunosuppressant drug azathioprine (50 to 100 $\mathrm{mg} /$ day) or levamisole (150 mg/day). ${ }^{22,23}$

Other treatment modalities include Calcineurin inhibitor, Retinoids, Dapsone, Hydroxycholroquin, mycophenolate mofetil and enoxaparin.

\begin{tabular}{|l|c|c|c|}
\hline \multicolumn{1}{|c|}{ Corticosteroids } & Retinoids & $\begin{array}{c}\text { Immunosuppresive } \\
\text { agents }\end{array}$ & Others \\
\hline Topical & Topical & Azathioprine \\
Betamethasone valerate & Fenretinide & Cyclosporin & $\begin{array}{c}\text { Amphotericin A } \\
\text { Basiliximab }\end{array}$ \\
Clobetasol propionate & Isotretinoin & & $\begin{array}{c}\text { Diethyldithiocarbamate } \\
\text { Doxycycline } \\
\text { Fluocinolone Acetonide }\end{array}$ \\
Fluocinonide & Tretinoin & & Enoxaparin \\
Fluticasone propionate & & & Glycyrrhizin \\
Hydrocortisone & & & Griseofulvin \\
hemisuccinate & & & Hydroxychloroquine sulphate \\
Triamcinolone Acetonide & & Interferon \\
Betamethasone Phosphate & & Immuno & Non Pharmacological T/T \\
Systemic & Pystemic & Podulator Drugs & Reflexotherapy \\
Prednisone & Etretinate & Levamisole & Surgery \\
Methylprednisolone & Isotretinoin & Dapsone & Psychotherapy \\
& Temarotene & Tacrolimus & Photopheresis \\
\hline
\end{tabular}

\section{Emperical treatment for oral lichen planus ${ }^{21}$}

Tacrolimus: A number of studies have been conducted regarding the role of topical application of $0.1 \%$ Tacrolimus particularly in refractory cases of OLP. Tacrolimus is a macrolide immunosuppressant. It has a greater mucosal penetration and is more effective than other immunosuppressants used topically. Some studies have proved that tacrolimus has a tendency to provide a better long-term pain reduction than topical corticosteroid. Although the adverse effects of topical tacrolimus use in OLP are typically local and benign for example burning sensation in the affected area. The long-term safety of this drug remains to be established. ${ }^{28-30}$

Griseofulvin is sometimes used in erosive OLP lesions, particularly in chronic and resistant cases where steroids are contraindicated.

UV Radiation: PUVA with methoxypsoaralen psoaralens modulates the function of cells of the immune system. ${ }^{24}$ Photodynamic therapy a photo sensitizing compound like methylene blue which is activated at a specific wavelength of laser light. PDT has immunomodulatory properties which may induce apoptosis in the hyperproliferating inflammatory cells. Thus it may be helpful in providing symptomatic relief in recalcitrant cases.

Curcumin in higher doses have also been tried to provide symptomatic relief in OLP patients. ${ }^{31}$

Surgical management Cryosurgery and carbon dioxide laser therapies have been tried in management of OLP lesions but they are not so useful for erosive and atropic type. ${ }^{21,25,26}$

Natural Alternatives: Lycopene and green tea (epigallocatechin-3-gallate) are potent antioxidants. ${ }^{27}$

An understanding of pathogenesis of OLP has lead to many studies dealing with blocking IL-2, IFN I $_{\gamma}$ INF $\alpha$, RANTES or MMP-9 activity and up-regulating $b$ TGB- $\beta$ activity. These studies have opened the doors of future treatment options in OLP. ${ }^{21}$ 


\section{Conclusion}

In the present case report we have explored the use of local drug delivery system for treatment of desquamative gingivitis in OLP using $10 \mathrm{mg}$ crushed prednisolone tablets. This can be an effective treatment modality in such chronic cases. The topical steroid application is preferred as there are fewer side effects than with systemic administration. Although long term application of topical steroids may lead to secondary candidiasis, thinning of the oral mucosa and discomfort or burning sensation on application. Prolonged use of potent topical corticosteroids with occlusal dressing can also cause adrenal suppression.

Thus there is no water tight management therapy in oral lichen planus, it needs to be customized according to each patient and should include changes in lifestyle and stress management. Innovations in management protocols are essential due to the chronic and persistent nature of disease and also increasing incidence of malignant transformation.

\section{Conflict of Interest: None declared.}

\section{References}

1. McCarthy. L.P. Diseases of the oral mucosa. $2^{\text {nd }} e d$. Autoimmune collagen disorders.1982:225-345.

2. Petrou- Amerikanou C, Markopoulos AK. Prevalence of oral lichen planus in diabetes mellitus according to the type of diabetes. Oral Disease. 1998;4:37-40.

3. Ongole. R Praveen B.N. Textbook of Oral Medicine and Radiology. $2^{\text {nd }}$ ED. Autoimmune Disorders. 2013:590605.

4. Davidson. Principles and practice of medicine $19^{\text {th }}$ ED. Churchill Livingstone, 2002: 642- 682.

5. Wilson E. Journal of cutaneous medicine, 1869;3:120335.

6. Shafer. Hine. Levy. Shafer's textbook of Oral Pathology. $6^{\text {th }}$ ED. Elsevier, 2009: 808.

7. Greenburg. Glick. Ship; Burket's Oral Medicine, $11^{\text {th }}$ ED. BC Decker, 2008:90.

8. Scully C, Carrozzo M. Oral mucosal disease: Lichen planus. Br J Oral Maxillofac Surg. 2008;46:15-21.

9. James Guggenheimer, Paul A. Moore. Insulin dependent diabetes mellitus and oral soft tissue pathologies. Oral Med Oral Pathol Oral Radiol Endod. 2000.

10. Andreasen JO. Oral lichen planus. 1. A clinical evaluation of 115 cases. Oral Surg Oral Med Oral Pathol. 1968;25:31-42.

11. Mc Carthy PL, Shklar G. 2nd ed. Philadelphia: Lea and Febiger; 1980. Diseases of the Oral mucosa.

12. Maryam Rad. Correlation between clinical and histopathologic diagnoses of oral lichen planus based on modified WHO diagnostic criteria. Oral Surg Oral Pathol Oral Radiol Endod. 2009;107:796-800.

13. Lodi Giovanni. Current controversies in oral lichen planus: Report of an international consensus meeting. Part 2. Clinical management and malignant transformation. Oral Surg Oral Med Oral Pathol Oral Radiol Endod. 2005;100:164-78.

14. Shafer. Hine. Levy. Shafer's textbook of Oral Pathology. $6^{\text {th }}$ ED. Elsevier, 2009:823-824.

15. Neville BW, Damm DD, Allen CM, Bouquot JE. Dermatological diseases. In: Neville BW, Damm DD, Allen CM, Bouquot JE, editors. Oral and Maxillofacial
Pathology. 2nd ed. Philadelphia: Saunders; 2002. pp. 680-5.

16. Greenberg Martin s., Glick Michael. Oral Medicine Diagnosis \& Treatment. Burket's Tenth Edition. 2003:133.

17. Peng Shi, Wei Liu. Podoplanin and ABCG2: Malignant Transformation Risk Markers for Oral Lichen Planus. Cancer Epidemiol Biomarkers Prev. 2010;19:844-849.

18. D Eisen, M Carrozzo. Oral lichen planus: clinical features and management. Oral Diseases. 2005;11:338-349.

19. PB Sugerman, NW Savage. Oral lichen planus: Causes, diagnosis and management. Australian Dental Journal. 2002;47:(4):290-297.

20. Huber Michaell A. Oral Lichen planus: Quintessence int. 2004;35:731-752.

21. Lavanya N, Jayanthi P. Oral lichen planus: An update on pathogenesis and Treatment. Journal of Oral and Maxillofacial Pathology. 2011(15):2.

22. Ahadian H, Akhavan Karbassi MH, Vahidi AR, Owlia F. Comparison of two corticosteroids mouthwashes in treatment of symptomatic oral lichen planus. J Dent Shiraz Univ Med Sci. 2012;13:49-53.

23. Gujjar. Treatment Modalities of Oral Lichen Planus with an Update IJSS Case Reports \& Reviews. 2015;2(4).

24. Kini R, Nagaratna DV, Saha A. Therapeutic management of oral lichen planus: A review for the clinicians. World $J$ Dent. 2011;2:249-53.

25. Kvaal SI, Angell-Petersen E, Warloe T. Photodynamic treatment of oral lichen planus. Oral Surg Oral Med Oral Pathol Oral Radiol. 2013;115:62-70.

26. Aghahosseini F, Arbabi-Kalati F, Fashtami LA, Fateh M, Djavid GE. Treatment of oral lichen planus with photodynamic therapy mediated methylene blue: A case report. Med Oral Patol Oral Cir Bucal. 2006;11:E126-9.

27. Patil S, Khandelwal S, Sinha N, Kaswan S, Tipu FR. Treatment modalities of oral lichen planus: An update. $J$ Oral Diagn. 2012;1:47-52.

28. Azizi A \& Lawaf S. The comparison of efficacy of adcortyl ointment and topical tacrolimus in treatment of erosive oral lichen planus. J Dent Res Dent Clin Dent Prospects. 2007;1(3):99-102.

29. Radfar L, Wild RC \& Suresh L (2008) A comparative treatment study of topical tacrolimus and clobetasol in oral lichen planus. Oral Surg Oral Med Oral Pathol Oral Radiol Endod. 105(2):187-193.

30. Sonthalia S \& Singal A. Comparative efficacy of tacrolimus $0.1 \%$ ointment and clobetasol propionate $0.05 \%$ ointment in oral lichen planus: a randomized double-blind trial. Int J Dermatol. 2012;51(11):13711378.

31. Chainani-Wu N, Collins K, Silverman S Jr. Use of curcuminoids in a cohort of patients with oral lichen planus, an autoimmune disease. Phytomedicine. 2012;19(5):418-423. 